\title{
Effect of pressure on the electrical transport and structure of $\mathrm{TiOCl}$
}

\author{
Martin K. Forthaus, ${ }^{1}$ Timo Taetz, ${ }^{2}$ Angela Möller, ${ }^{2}$ and Mohsen M. Abd-Elmeguid ${ }^{1}$ \\ ${ }^{1}$ II. Physikalisches Institut, Universität zu Köln, Zülpicher Strasse 77, D-50937 Köln, Germany \\ ${ }^{2}$ Institut für Anorganische Chemie, Universität zu Köln, Greinstrasse 6, D-50939 Köln, Germany
}

(Received 18 January 2008; revised manuscript received 14 March 2008; published 15 April 2008)

\begin{abstract}
We have investigated the effect of pressure on the electrical transport and the structural properties of TiOCl. The temperature dependence of the electrical resistivity up to $24 \mathrm{GPa}$ shows no indication of an insulator-tometal transition. However, the analysis of the pressure dependence of the energy gap $\left(E_{g}\right)$ around $300 \mathrm{~K}$ reveals first a strong decrease of $E_{g}$ with increasing pressure, followed by a much weaker decrease of $E_{g}$ above $13 \mathrm{GPa}$, indicating a change of the electronic structure. The analysis of the pressure dependence of the lattice parameters up to $18 \mathrm{GPa}$ reveals an extreme anisotropic and anomalous change of the lattice parameters with increasing pressure. While the lattice parameters $b$ and $c$ smoothly decrease up to the highest pressure, the lattice parameter $a$ remains nearly pressure independent above about $11 \mathrm{GPa}$. It is shown that such anomalous change of the lattice parameters with pressure strongly affects the relative strength of the effective direct and indirect hopping along the $b$ and $a$ axis, respectively, and therefore it is responsible for the observed pressureinduced change of the electronic properties of $\mathrm{TiOCl}$ above $13 \mathrm{GPa}$.
\end{abstract}

DOI: $10.1103 /$ PhysRevB.77.165121

PACS number(s): 72.20.-i, 71.30.+h, 62.50.-p

\section{INTRODUCTION}

Low-dimensional spin $1 / 2$ systems such as transition metal compounds have attracted much interest due to their interesting novel ground states. This is related to the fact that such unusual ground states are driven by a complex interplay between charge, orbital, spin, and lattice degrees of freedom. A coupling of a one-dimensional antiferromagnetic $S=1 / 2$ chain with the lattice may result in a spin-Peierls transition with a nonmagnetic (singlet) dimerized ground state. $\mathrm{CuGeO}_{3}$ is the first well-established example for such a transition. ${ }^{1-3}$ More exciting phenomena are expected if the spins are additionally coupled to the orbital or charge degree of freedom. An exemplary system to study such an interplay is the layered quasi-one-dimensional Mott insulator TiOCl $\left(\mathrm{Ti}^{3+}, 3 d^{1}\right)$ which recently has been the subject of intense research. ${ }^{5,6,4,7-11}$

$\mathrm{TiOCl}$ crystallizes in an orthorhombic quasi-twodimensional structure (FeOCl-type) where buckled Ti-O bilayers within the $a b$ plane are well-separated by $\mathrm{Cl}^{-}$ions (see Fig. 1). This results in an orbital ordering ${ }^{4}$ with formation of quasi-1D $S=1 / 2$ Heisenberg chains along the $b$ axis. The strong coupling of the 1D antiferromagnetic chains to the lattice at low temperature leads to an unconventional spinPeierls transition $\left(T_{c 1}=67 \mathrm{~K}\right),{ }^{5}$ which is manifested by a sharp decrease of the magnetic susceptibility below $T_{c 1}$, a corresponding doubling of the unit cell along the $b$ axis, ${ }^{6}$ and the existence of a spin excitation gap as observed, e.g., in electron spin resonance $(\mathrm{ESR})^{4}$ and $\mathrm{NMR}^{7}$ experiments. Moreover, a second-order phase transition was observed at $T_{c 2}=94 \mathrm{~K}$ which is suggested to be triggered by the interchain interactions in the bilayer structure. ${ }^{9,10}$

Among the variety of the experimental techniques being appropriate to explore the ground state properties of $\mathrm{TiOCl}$, application of external pressure can be used to tune the coupling between the lattice and electronic degrees of freedom and thereby provides information about their interplay. In this respect, $\mathrm{TiOCl}$ as a Mott insulator is a promising candi- date for investigating the pressure-induced metal insulator transition (MIT). Indeed, recently the possibility of a pressure-induced insulator-to-metal transition in $\mathrm{TiOCl}$ has been reported from high pressure infrared spectroscopy. Here, the strong suppression of the transmittance and the abrupt decrease of the near infrared reflectance above $12 \mathrm{GPa}$ were attributed to be an indicator of a pressureinduced insulator metal transition. ${ }^{11}$

Motivated by the aspects mentioned above and in view of the possibility of achieving a superconducting state in $\mathrm{TiOCl}$ as observed in low-dimensional systems, e.g., $\mathrm{Sr}_{2.5} \mathrm{Ca}_{11.5} \mathrm{Cu}_{24} \mathrm{O}_{41}$ (Ref. 12) and $\beta-\mathrm{Na}_{0.33} \mathrm{~V}_{2} \mathrm{O}_{5},{ }^{13}$ we have investigated the effect of pressure on the electrical transport and structural properties of $\mathrm{TiOCl}$.

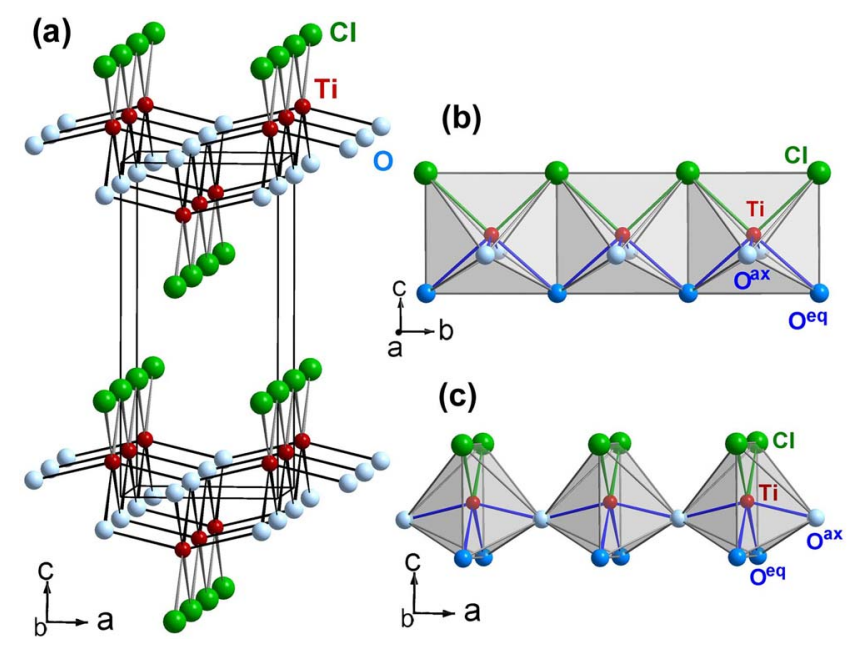

FIG. 1. (Color online) Perspective view of the crystal structure of $\mathrm{TiOCl}$ (a). A representation of the one-dimensional antiferromagnetic $S=1 / 2$ coupled chain (b) and the connectivity along the $a$ axis (c) are given as well as the labeling for a distorted octahedron $\left[\mathrm{TiO}_{2}^{\mathrm{ax}} \mathrm{O}_{2}^{\mathrm{eq}} \mathrm{Cl}_{2}\right]$. 


\section{EXPERIMENT}

Single crystals of $\mathrm{TiOCl}$ have been prepared according to the method described in Ref. 14. Samples (ground crystals from chemical vapor transport experiment) checked by x-ray diffraction were single phase (orthorhombic, space group Pmmn). The pressure dependence of the electrical resistance between 1.6 and $300 \mathrm{~K}$ and up to $24 \mathrm{GPa}$ has been measured using the four-point technique in a diamond anvil cell (DAC) made from a special Ti alloy which ensures thermal stability against temperature variations (constant pressure). The metallic gasket (Inconel 750) of the DAC was insulated from the four leads by thin $(12 \mu \mathrm{m})$ capton foil and a mixture of epoxy and $\mathrm{Al}_{2} \mathrm{O}_{3}$ serving also as a pressure transmitting medium. The resulting sample chamber had a diameter and a height of $\sim 100$ and $\sim 50 \mu \mathrm{m}$, respectively. The distance of the leads determining the voltage drop on the sample was about $50 \mu \mathrm{m}$. The pressure gradient within the pressure cavity was between $5 \%$ and $7 \%$. Pressure was measured by the ruby luminescence method. ${ }^{15,16}$ Data were taken in a ${ }^{4} \mathrm{He}$ bath cryostat using dc current and by measuring the voltage drop for both polarities. The measurements of the lattice parameters as a function of pressure were performed by energy dispersive X-ray diffraction (EDX) at the Hamburger Synchrotronstrahlungslabor (HASYLAB), beamline F3 using the DAC (same type) technique up to about $20 \mathrm{GPa}$ at $300 \mathrm{~K}$. A standard 4:1 methanol-ethanol mixture was used as a pressure transmission medium and the pressure was determined by the ruby luminescence as in electrical resistivity measurements. In addition, accurate EDX on the sample in the pressure range up to $8 \mathrm{GPa}$ has been performed using the multi anvil x-ray device (MAX 80), beamline F2.1 at HASYLAB. In this case the sample chamber consists of a small boron nitride container with an internal diameter of $\sim 1 \mathrm{~mm}$ which at the same time acts as a pressure transmission medium. The cubic sample chamber is uniformly compressed by six anvils in a large hydraulic press. The pressure is determined from the change of the lattice parameter of $\mathrm{NaCl}$ using the Decker equation of state. ${ }^{17}$

\section{RESULTS AND DISCUSSION}

In the following, we first present and discuss the pressure dependence of the electrical resistivity of $\mathrm{TiOCl}$ and the related electronic phase transition. We then consider the variation of the structural parameters under high pressure. Finally, we discuss the driving mechanism of the pressure-induced change of the electronic state.

\section{A. Effect of pressure on the electrical transport}

Figure 2 displays the temperature dependence (logarithmic scale) of the electrical resistance $R(T, p)$ of $\mathrm{TiOCl}$ at different pressures up to $24.2 \mathrm{GPa}$. The $R(T, p)$ curves exhibit a similar feature: as shown in Fig. 2, we find a dramatic reduction of the value of $R(294 \mathrm{~K}, p)$ with increasing pressure by more than six orders of magnitude, corresponding to a decrease of the specific resistivity $\rho$ from $\rho \sim 10^{5} \Omega \mathrm{cm}$ (at $6.3 \mathrm{GPa}$ ) to $\rho \sim 10^{-1} \Omega \mathrm{cm}$ (at $24.2 \mathrm{GPa}$ ). However, as evident from the temperature dependence of the $R(T, p)$ curves

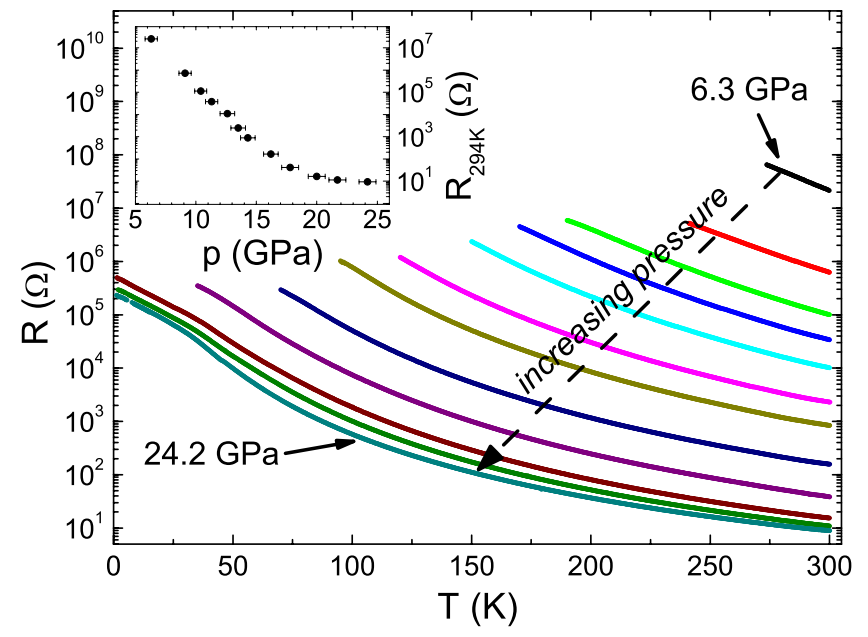

FIG. 2. (Color online) Electrical resistance of TiOCl for various pressures $(6.3<p<24.2 \mathrm{GPa})$ in the range $1.6<T<300 \mathrm{~K}$ in a logarithmic plot. The inset shows the pressure dependence of the electrical resistance at $T=294 \mathrm{~K}$.

$(d R / d T<0)$, the sample remains insulating up to the highest pressure of $24.2 \mathrm{GPa}$. The observation of an insulating state up to $24 \mathrm{GPa}$ is in contrast to that reported from high pressure infrared measurements onTiOCl which have been described as a possible pressure-induced MIT above $12 \mathrm{GPa}^{11}$

To obtain further information about the pressure-induced variation of the electrical transport in $\mathrm{TiOCl}$ and its possible relevance to the high pressure infrared data, we have performed a rough estimation of the change in the activation energy $E_{g}$ as a function of pressure $\left[E_{g}(p)\right]$. Due to the extremely high resistance $\left(R \sim 10^{8} \Omega\right)$, we only consider the $R(T, p)$ data above $6 \mathrm{GPa}$ and in a limited temperature range $(270<T<300 \mathrm{~K})$. The estimated values of $E_{g}(p)$ as obtained from an Arrhenius plot are shown in Fig. 3. Despite the much lower absolute values of $E_{g}(p)$ compared to those obtained from optical spectroscopy, the pressure dependence of $E_{g}, \partial E_{g} / \partial p$, reveals an abrupt change at about $13 \mathrm{GPa}$ where $\partial E_{g} / \partial p$ decreases by a factor of $\approx 4\left(\partial E_{g} / \partial p=\right.$

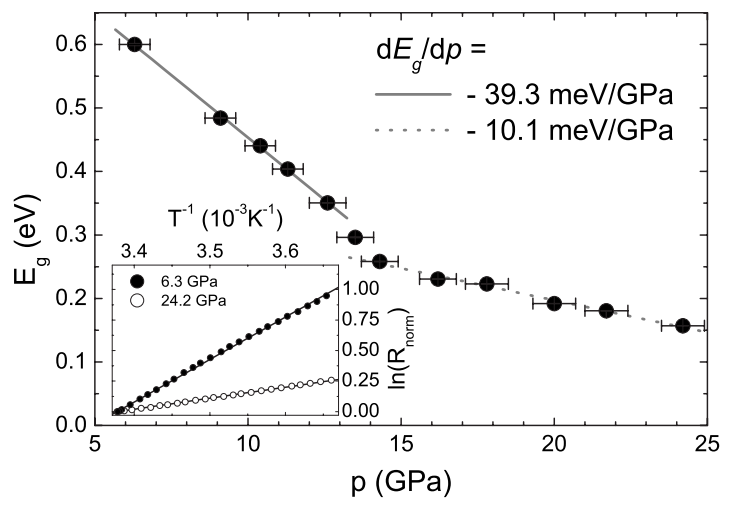

FIG. 3. Pressure dependence of the activation energy $E_{g}(p)$ around $300 \mathrm{~K}$ obtained from the temperature dependence of the electrical resistance shown in Fig. 2 (see text). Inset shows the Arrhenius plot using the normalized resistance $\left[R_{\text {norm }}=R(T) / R(294 \mathrm{~K})\right]$ for $6.3 \mathrm{GPa}(\bullet)$ and $24.2 \mathrm{GPa}(\circ)$ in the range $270<T<300 \mathrm{~K}$; solid lines are linear fits. 


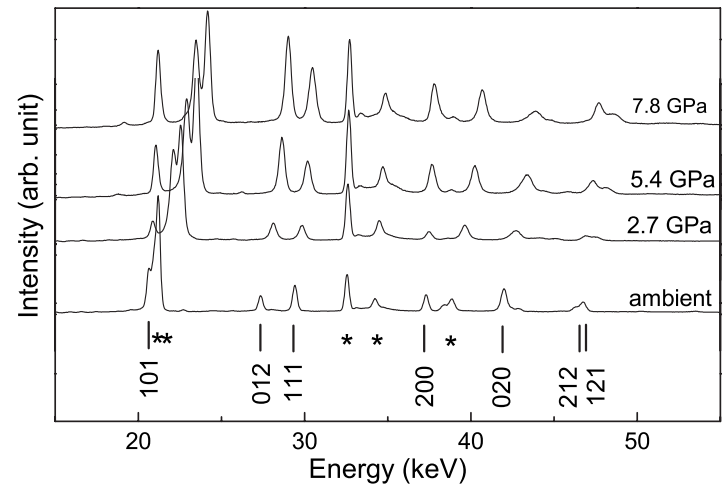

FIG. 4. Typical diffraction patterns for selected pressures up to 7.8 GPa measured at beamline F2.1, HASYLAB. Asterisks mark the peaks that result from boron nitride (see text).

$-39 \mathrm{meV} / \mathrm{GPa}$ and $-10 \mathrm{meV} / \mathrm{GPa}$ for $6<p<13 \mathrm{GPa}$ and $13<p<24 \mathrm{GPa}$, respectively).

This finding clearly indicates a change of the electronic structure of TiOCl above $13 \mathrm{GPa}$. As a matter of fact, our observation of such a pressure-induced change of the electronic structure is at nearly the same critical pressure at which the high pressure infrared data suggest a possible pressure-induced insulator-to-metal transition. Thus the two experimental techniques detect a clear change of the electronic structure of $\mathrm{TiOCl}$ at about $13 \mathrm{GPa}$. However, a value of $E_{g} \sim 0.3 \mathrm{eV}$ at $13 \mathrm{GPa}$ clearly indicates a semiconducting state at $300 \mathrm{~K}$. Moreover, we find a very high value of $\rho$ of about $\rho \sim 10^{-1} \Omega \mathrm{cm}$ at $300 \mathrm{~K}$ which dramatically increases by more than four orders of magnitude upon decreasing temperature, typical for a semiconducting state.

In this context, we would like to point out that the existence of a semiconducting gap of $\sim 0.3 \mathrm{eV}$ at $13 \mathrm{GPa}$ cannot be detected by infrared measurements since the lowest energy used was about $0.25 \mathrm{eV} .{ }^{11}$

\section{B. Effect of pressure on the crystal structure}

In order to provide an answer to the question regarding the driving mechanism and/or the origin of the observed pressure-induced change of the electronic state above $12 \mathrm{GPa}$, we now consider the pressure dependence of the

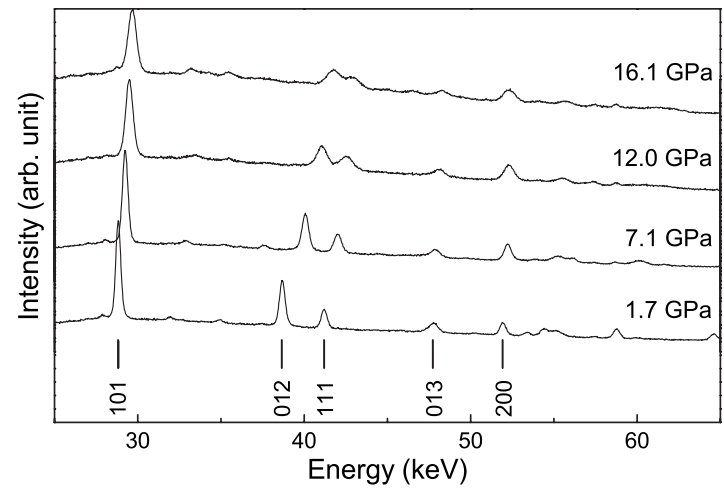

FIG. 5. Typical diffraction patterns for selected pressures up to $18 \mathrm{GPa}$ measured at beamline F3, HASYLAB.

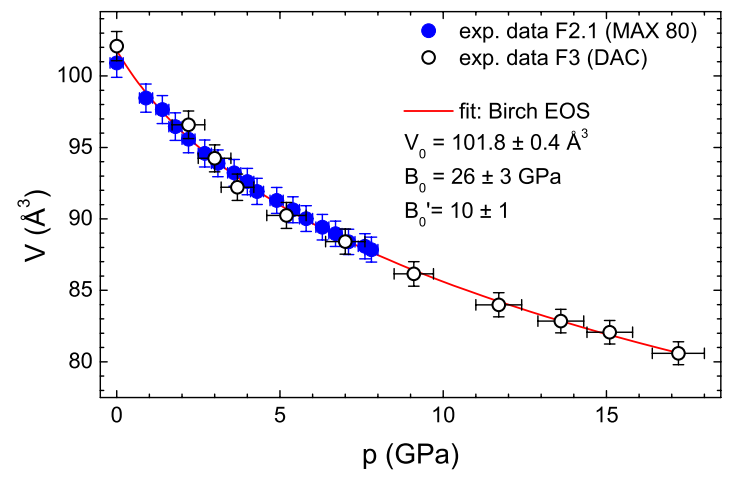

FIG. 6. (Color online) Pressure dependence of the unit cell volume of $\mathrm{TiOCl}$ at $300 \mathrm{~K}$. Blue and black data points denote the measurements done up to 8 and $18 \mathrm{GPa}$, respectively. The solid line through the data points is a fit using Birch's equation.

lattice parameters of TiOCl. Structural data were collected at beamlines F2.1 and F3 at HASYLAB for pressures up to 8 and $18 \mathrm{GPa}$, respectively. Figures 4 and 5 show typical energy dispersive diffraction patterns recorded at $300 \mathrm{~K}$.

All sample peaks could be indexed according to the orthorhombic FeOCl-type structure (Pmmn) up to $18 \mathrm{GPa}$. We find no evidence for a structural phase transition within the accuracy of our measurements. We also find after releasing the pressure no change of the FeOCl-type structure which exclude a pressure-induced decomposition of the sample. The pressure-volume relationship of $\mathrm{TiOCl}$ up to $18 \mathrm{GPa}$ including both experimental data sets up to 8 and $18 \mathrm{GPa}$ are plotted in Fig. 6. The data reveal a smooth variation of the volume with pressure which can be described by Birch's equation ${ }^{18}$ with bulk modulus $B_{0}=26 \pm 3 \mathrm{GPa}$ and its pressure derivative $B_{0}^{\prime}=10 \pm 1$.

However, as shown in Figs. 7 and 8, the variation of the lattice parameters $a, b$, and $c$ of the unit cell is extremely anisotropic. First, we discuss the pressure dependence of $a$, $b$, and $c$ up to $8 \mathrm{GPa}$. A fit to the data using Birch's equation results in the following values of the inverse of the linear compressibility $B_{x}(x=a, b, c)$ and its pressure derivative $B_{x}^{\prime}$ along the $a, b$, and $c$ axis, respectively: $B_{a}=445 \pm 40 \mathrm{GPa}$ $\left(B_{a}^{\prime}=19 \pm 9\right), \quad B_{b}=145 \pm 7 \mathrm{GPa} \quad\left(B_{b}^{\prime}=10 \pm 2\right), \quad$ and $\quad B_{c}$ $=30 \pm 7 \mathrm{GPa}\left(B_{c}^{\prime}=40 \pm 10\right)$.

These values reflect an extremely large anisotropic compression of the lattice parameters. The compression along the

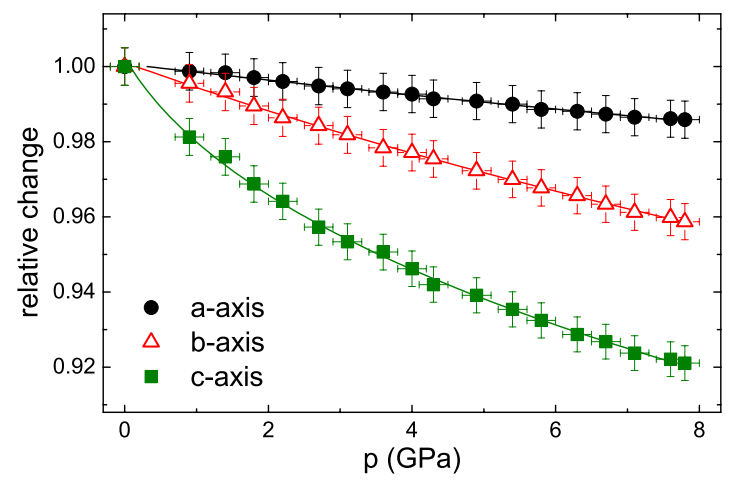

FIG. 7. (Color online) Relative change of the $a, b$, and $c$ axes of the orthorhombic unit cell of $\mathrm{TiOCl}$ at $300 \mathrm{~K}$ with pressure. 


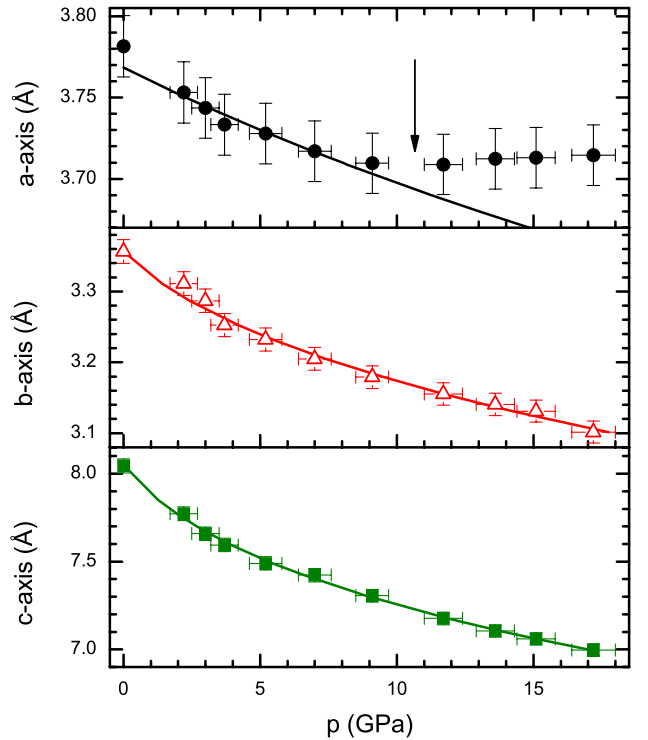

FIG. 8. (Color online) Pressure dependence of the lattice parameters $(a, b$, and $c)$ of $\mathrm{TiOCl}$ at $300 \mathrm{~K}$ up to $18 \mathrm{GPa}$. The arrow in (a) marks the pressure-induced anomalous change of the $a$ axis above $11 \mathrm{GPa}$.

$c$ axis (i.e., between the $\mathrm{Cl}^{-}$layers) is about five times larger than that along the $b$ axis (i.e., along the $\mathrm{Ti}^{3+}$ chains) and even 15 times larger than that along the $a$ axis. Such a large anisotropic compression points to a structural instability at higher pressures. This is indeed what we have observed [see Figs. 8(a)-8(c)]. By increasing pressure above $11 \mathrm{GPa}$, we find the following unusual behavior: while the $b$ and $c$ axis decrease smoothly with pressure up to $18 \mathrm{GPa}$, the $a$ axis remains nearly constant above $\sim 11 \mathrm{GPa}$. As we will show in Sec. III C, this anomalous behavior is strongly connected with the observed pressure-induced change of the electrical transport in $\mathrm{TiOCl}$.

Despite the fact that our EDX data does not allow a direct determination of the Ti-O bond distances as, e.g., from angle-dispersive diffraction experiments, we show in a simple model how the Ti-O distances change with pressure. The observed large anisotropic compression of the lattice parameters can be related to differences in the chemical bondings within the $\mathrm{TiOCl}$ structure. The pressure dependence of the $a$ axis reflects the reduction of Ti-O distances. In the ionic limit a total decrease of $0.08 \AA$ along the $a$ axis [see Fig. 8(a)] leads, due to the O-Ti-O angle, to a decrease of about $0.05 \AA$ at $11 \mathrm{GPa}$ from $1.96 \AA(0 \mathrm{GPa})$ to $1.91 \AA$ and 2.20 to $2.14 \AA$ for $d\left(\mathrm{Ti}-\mathrm{O}^{\mathrm{ax}}\right)$ and $d\left(\mathrm{Ti}^{-} \mathrm{O}^{\mathrm{eq}}\right)$, respectively; assuming that the two $\mathrm{Ti}-\mathrm{O}$ bonds exhibit the same pressure dependence. The average value of $2.02 \AA$ for the $d(\mathrm{Ti}-\mathrm{O})$ value is in agreement with the ionic lower limit of $2.05 \AA$ at $0 \mathrm{GPa}$ derived from the ionic radii of the respective ions $\left(\mathrm{Ti}^{3+}\right.$ and $\left.\mathrm{O}^{2-}\right) .{ }^{19}$ Furthermore, pressure studies of $\mathrm{YTiO}_{3}$ support this assignment of structural details with the observation of an average $d(\mathrm{Ti}-\mathrm{O})$ distance of $2.02 \AA$ at $11 \mathrm{GPa}^{20}$ It is obvious that no further significant decrease in the Ti-O distance can be anticipated and therefore it is expected that the decrease of the $a$ axis levels off above 11 GPa (see Fig. 9 and for structural details see Fig. 1). The large pressure

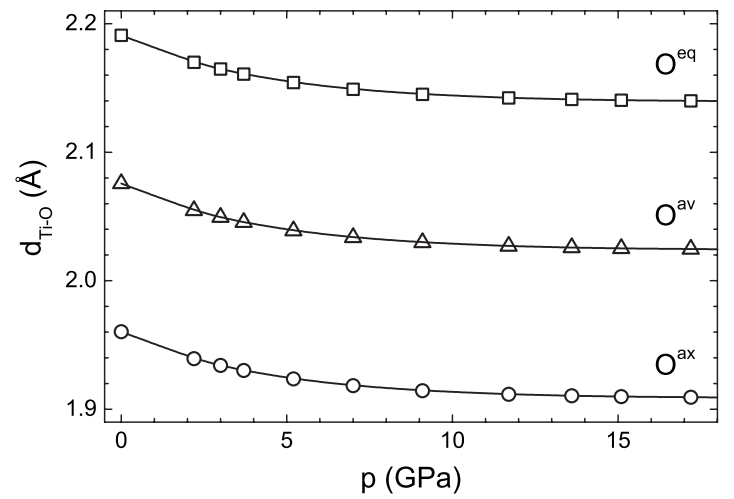

FIG. 9. Pressure dependence of the respective Ti-O distances as derived from the lattice parameter $a$ in comparison with the average value of $\mathrm{Ti}-\mathrm{O}^{\text {av }}$.

dependence of the $c$ axis ( $\mathrm{Cl}-\mathrm{Cl}$ interlayer distances) is related to the highly compressible weak van der Waals interaction [see Fig. 8(c)].

Also the moderate decrease of the value of the lattice parameter $b$ with increasing pressure directly reflects the corresponding change of the Ti-Ti distance in the TiOCl type of structure. At the highest pressure (18 GPa) the value of the Ti-Ti distance amounts to $3.10 \AA$. This value is still larger than that necessary for the formation of $\mathrm{Ti}-\mathrm{Ti}$ metallic bonds, since in Ti metal the nearest-neighbor bond distance between Ti atoms is $\sim 2.89 \AA$ and thus is consistent with the observed semiconducting behavior at higher pressures.

\section{Structural instability versus electrical transport}

Finally, we want to show that the anomalous behavior of the pressure dependence of the lattice parameter $a$ above $11 \mathrm{GPa}$ [see Fig. 8(a)] is the key for understanding the pressure-induced change of the electronic properties of $\mathrm{TiOCl}$ at $300 \mathrm{~K}$. The abrupt decrease of the pressure dependence of $E_{g}$ is observed at a pressure higher than that of the anomalous change of the lattice parameter $a$. This can be related to the fact that the electrical transport is not expected to be very sensitive to a small structural distortion along the $a$ axis, since it reflects the average change of the electrical transport along the whole crystal lattice. In addition, x-ray diffraction and electrical resistivity measurements were performed using the same type of the DAC but with different pressure media which can affect the value of the critical pressure.

Since TiOCl is a Mott insulator, we assume that the electrical conduction is due to electron hopping. This is related to the fact that for such systems the average interaction energy between electrons (Coulomb energy $U$ ) is very large compared to the effective bandwidth $(W)$. As a result, the electrons are localized at each site and the conductivity at finite temperatures occurs by electron hopping between neighboring sites, i.e., hopping conductivity. Accordingly, the number of carriers $(n)$ at a given temperature $n(T)$ is determined by the Boltzmann's statistics $\left[n(T) \sim e^{-E_{g} /\left(k_{\mathrm{B}} T\right)}\right]$, the electrical conductivity follows exponential activation. The layered structure of TiOCl (see Fig. 1) contains two different types 
of $\mathrm{Ti}^{3+}$ chains which can contribute to the electrical transport. This can take place via two routes: (i) indirect hopping between Ti $3 d$ electrons via oxygen $2 p$ along the $a$ axis; and (ii) direct hopping (overlap) between the $\mathrm{Ti} 3 d$ orbitals along the $b$ axis. Thus the electrical conduction in $\mathrm{TiOCl}$ and its variation with pressure is intimately connected with the pressure-induced variation of the lattice parameters $a$ and $b$. The effective hopping in TiOCl can be described by the hopping integrals $t_{d d}$ for the direct Ti-Ti hopping and $t_{p d}$ for the indirect Ti-O-Ti hopping along the $b$ and $a$ axes, respectively. Both hopping integrals are sensitive to the variation of the nearest-neighbor distance between Ti-Ti $\left(d_{\mathrm{Ti}-\mathrm{Ti}}\right)$ and Ti-O $\left(d_{\text {Ti-O }}\right)$, respectively.

However, according to Ref. $21, t_{d d}$ scales with $d^{-5}$ whereas the total effective $p d$-hopping $\left(\left\langle t_{p d}\right\rangle=2 t_{p d}\right)$ scales with $d^{-7}$. Accordingly, the pressure dependence of the effective hopping is given by $\partial t_{d d} / \partial p \sim \frac{5}{d^{6}} \Delta d_{\mathrm{Ti}-\mathrm{Ti}}$ and $\partial t_{p d} / \partial p$ $\sim \frac{7}{d^{8}} \Delta d_{\mathrm{Ti}-\mathrm{O}}$ where $\Delta d_{\mathrm{Ti}-\mathrm{Ti}}$ and $\Delta d_{\mathrm{Ti}-\mathrm{O}}$ are the pressure-induced changes of the Ti-Ti and Ti-O nearest-neighbor distance, respectively. This simple estimation clearly indicates that the indirect (Ti-O-Ti) hopping is much more sensitive to pressure than the direct (Ti-Ti) hopping. Obviously, such a very high sensitivity of the indirect hopping along the $a$ axis to $d_{\mathrm{Ti}-\mathrm{O}}$ dominates the pressure dependence of the electrical conductivity and consequently that of $E_{g}(p)$ in TiOCl.

Recalling now the observed anomalous compression of $a$ above $11 \mathrm{GPa}$ compared to the smooth decrease of $b$, we can explain the abrupt decrease of the pressure dependence of $E_{g}(p)$ above $13 \mathrm{GPa}$ in the following way: in the low pressure range $(p<13 \mathrm{GPa})$, both $a$ and $b$ axes (i.e., $d_{\text {Ti-O }}$ and $d_{\mathrm{Ti}-\mathrm{Ti}}$ ) smoothly decrease with increasing pressure and cause a corresponding increase of effective hopping $\left(t_{p d}\right.$ and $\left.t_{d d}\right)$ which in turn leads to a strong decrease of $E_{g}$ as pressure increases. Above $13 \mathrm{GPa}$, while $b$ further decreases with increasing pressure, $a$ remains almost pressure independent (blocked) up to $18 \mathrm{GPa}$. This implies a nearly constant value of $d_{\text {Ti-o }}$ with the consequence that the indirect hopping along the $a$ axis $\left\langle t_{p d}\right\rangle$ remains unchanged in this pressure range. In such a case, the pressure-induced change of $E_{g}(p)$ will be only governed by the weak pressure dependence of $t_{d d}$ (see above). This indicates that the observed abrupt decrease of the pressure dependence of $E_{g}(p)$ above $\sim 13 \mathrm{GPa}$ is driven by the anomalous change of the lattice parameter $a$ as a function of pressure. Moreover, our finding of a pressure independent value of the lattice parameter $a$ and consequently the effective hopping $\left\langle t_{p d}\right\rangle$ above $13 \mathrm{GPa}$ provides an explanation why $\mathrm{TiOCl}$ remains in a semiconducting state up to higher pressures of $\sim 24 \mathrm{GPa}$.

\section{SUMMARY}

In summary, we have studied the effect of pressure on the electrical transport and structural properties of the quasi-twodimensional $S=1 / 2$ system TiOCl. The analysis of the temperature dependence of the electrical resistivity as a function of pressure indicates that $\mathrm{TiOCl}$ remains in a semiconducting state up to a pressure of $24 \mathrm{GPa}$. However, the pressureinduced decrease of the energy gap $\left(E_{g}\right)$ reveals an abrupt change above $13 \mathrm{GPa}$. This indicates a change of the electronic structure of $\mathrm{TiOCl}$ at higher pressures. Our investigation of the structural properties under high pressure provides information on the driving mechanism of the observed state at high pressures: the decrease of the lattice parameters $(a, b$, and $c$ ) is shown to be extremely anisotropic. Most important is the observation of an anomalous change of the lattice parameter $a$ above $11 \mathrm{GPa}$ with respect to $b$ and $c$. We have shown that such an anomalous change of the lattice parameter $a$ strongly affects the relative strength of the indirect (Ti-O-Ti) and direct (Ti-Ti) hopping along the $a$ and $b$ axes, respectively, and thus is suggested to be the driving mechanism of the observed change of the electronic properties of $\mathrm{TiOCl}$ above $13 \mathrm{GPa}$.

\section{ACKNOWLEDGMENTS}

The authors would like to thank D. I. Khomskii, S. Streltsov, M. Grüninger, and M. Braden for fruitful discussions. The help of C. Lathe and M. Wehber situated at beamline F2.1, HASYLAB, regarding the operation of the MAX 80 device is acknowledged. This project was supported by Deutsche Forschungsgemeinschaft (DFG) via Grant No. SFB 608.
${ }^{1}$ M. Hase, I. Terasaki, and K. Uchinokura, Phys. Rev. Lett. 70, 3651 (1993).

${ }^{2}$ J. P. Pouget, L. P. Regnault, M. Ain, B. Hennion, J. P. Renard, P. Veillet, G. Dhalenne, and A. Revcolevschi, Phys. Rev. Lett. 72, 4037 (1994).

${ }^{3}$ K. Hirota, D. E. Cox, J. E. Lorenzo, G. Shirane, J. M. Tranquada, M. Hase, K. Uchinokura, H. Kojima, Y. Shibuya, and I. Tanaka, Phys. Rev. Lett. 73, 736 (1994).

${ }^{4}$ V. Kataev, J. Baier, A. Möller, L. Jongen, G. Meyer, and A. Freimuth, Phys. Rev. B 68, 140405(R) (2003).

${ }^{5}$ A. Seidel, C. A. Marianetti, F. C. Chou, G. Ceder, and P. A. Lee, Phys. Rev. B 67, 020405(R) (2003).

${ }^{6}$ M. Shaz, S. van Smaalen, L. Palatinus, M. Hoinkis, M. Klemm, S. Horn, and R. Claessen, Phys. Rev. B 71, 100405(R) (2005).
${ }^{7}$ T. Imai and F. C. Chou, arXiv:cond-mat/0301425.

${ }^{8}$ P. Lemmens, K. Y. Choi, G. Caimi, L. Degiorgi, N. N. Kovaleva, A. Seidel, and F. C. Chou, Phys. Rev. B 70, 134429 (2004); P. Lemmens, K. Y. Choi, R. Valentí, T. Saha-Dasgupta, E. Abel, Y. S. Lee, and F. C. Chou, New J. Phys. 7, 74 (2005).

${ }^{9}$ R. Rüickamp, J. Baier, M. Kriener, M. W. Haverkort, T. Lorenz, G. S. Uhrig, L. Jongen, A. Möller, G. Meyer, and M. Grüninger, Phys. Rev. Lett. 95, 097203 (2005).

${ }^{10}$ D. Fausti, T. T. A. Lummen, C. Angelescu, R. Macovez, J. Luzon, R. Broer, P. Rudolf, P. H. M. van Loosdrecht, N. Tristan, B. Büchner, S. van Smaalen, A. Möller, G. Meyer, and T. Taetz, Phys. Rev. B 75, 245114 (2007).

${ }^{11}$ C. A. Kuntscher, S. Frank, A. Pashkin, M. Hoinkis, M. Klemm, M. Sing, S. Horn, and R. Claessen, Phys. Rev. B 74, 184402 
(2006)

${ }^{12}$ T. Nagata, M. Uehara, J. Goto, J. Akimitsu, N. Motoyama, H. Eisaki, S. Uchida, H. Takahashi, T. Nakanishi, and N. Môri, Phys. Rev. Lett. 81, 1090 (1998).

${ }^{13}$ T. Yamauchi, Y. Ueda, and N. Môri, Phys. Rev. Lett. 89, 057002 (2002).

${ }^{14}$ H. Schäfer, F. Wartenpfuhl, and E. Weise, Z. Anorg. Allg. Chem. 295, 268 (1958).

${ }^{15}$ G. J. Piermarini, S. Block, J. D. Barnett, and R. A. Forman, J. Appl. Phys. 46, 2774 (1975).
${ }^{16}$ H. K. Mao, J. Xu, and P. M. Bell, J. Geophys. Res. 91, 4673 (1986).

${ }^{17}$ D. L. Decker, J. Appl. Phys. 42, 3239 (1971).

${ }^{18}$ F. Birch, Phys. Rev. 71, 809 (1947).

${ }^{19}$ R. D. Shannon, Acta Crystallogr., Sect. A: Cryst. Phys., Diffr., Theor. Gen. Crystallogr. 32, 751 (1976).

${ }^{20}$ I. Loa, X. Wang, K. Syassen, H. Roth, T. Lorenz, M. Hanfland, and Y.-L. Mathis, J. Phys.: Condens. Matter 19, 406223 (2007).

${ }^{21}$ W. A. Harrison, Electronic Structure and the Properties of Solids (Dover, New York, 1989), p. 476. 